\title{
Observational Evidence of Multiple Stellar Populations in Globular Clusters
}

\author{
Giampaolo Piotto ${ }^{1}$ \\ ${ }^{1}$ Dipartimento di Astronomia, Università di Padova, \\ vicolo dell'Osservatorio, 2, I-35122, Padova, Italy \\ email: giampaolo.piotto@unipd.it
}

\begin{abstract}
An increasing number of photometric observations of multiple stellar populations in Galactic globular clusters is seriously challenging the paradigm of GCs hosting single, simple stellar populations. These multiple populations manifest themselves in a split of different evolutionary sequences as observed in the cluster color-magnitude diagrams. In this paper we will summarize the observational scenario.
\end{abstract}

Keywords. Globular Clusters, Stellar Populations, Photometry, Astrometry

\section{Introduction}

Globular star clusters (GC) occupy a prominent role in modern astrophysics. They are among the oldest Population II objects for which accurate ages can be derived, hence they set an independent constraint on Cosmology. As such, they must have formed before the bulk of the parent galaxy, and then can provide insight into galaxy formation. Given the large number of stars in them, they represent the ideal laboratory for testing and calibrating stellar evolutionary models, as well as for (N-body) dynamical studies. Finally, being though consisting of basically coeval and chemically homogeneous stars, they have been used as ideal templates for "simple stellar populations" (SSP) with which to test and calibrate synthetic models of stellar populations, a critical tool for studying galaxies at low as well as at high redshift.

Color-magnitude diagrams (CMD) like that of NGC 6397 shown in Fig. 1 fully support the paradigma of GCs hosting simple stellar populations. However, there is a growing body of observational facts which challenge this traditional view. Since the eighties we know that GCs show a peculiar pattern in their chemical abundances (see Gratton el al. 2004 for a recent review). While they are generally homogenous insofar Fe-peak elements are considered, they often exhibit large anticorrelations between the abundances of $\mathrm{C}$ and $\mathrm{N}, \mathrm{Na}$ and $\mathrm{O}, \mathrm{Mg}$ and $\mathrm{Al}$. These anticorrelations are attributed to the presence at the stellar surfaces of a fraction of the GC stars of material which have undergone $\mathrm{H}$ burning at temperatures of a few ten millions K (Prantzos et al. 2007; less for the $\mathrm{C}$ and $\mathrm{N}$ anticorrelation). This pattern is peculiar to GC stars; field stars only show changes in $\mathrm{C}$ and $\mathrm{N}$ abundances expected from typical evolution of low mass stars (Gratton et al. 2000; Sweigart \& Mengel 1979; Charbonnel 1994); it is primordial, since it is observed in stars at all evolutionary phases (Gratton et al. 2001); and the whole stars are interested (Cohen et al. 2002).

In addition, since the sixties (Sandage and Wildey 1967, van den Bergh 1967), we know that the horizontal branches (HB) of some GCs can be rather peculiar. In some GCs the HB can be extended to very hot temperatures implying the loss of most of the stellar envelope (see compilation by Recio-Blanco et al. 2006); the distribution of the stars along the HB can be clumpy, with the presence of one or more gaps (Ferraro et al. 1998, Piotto 


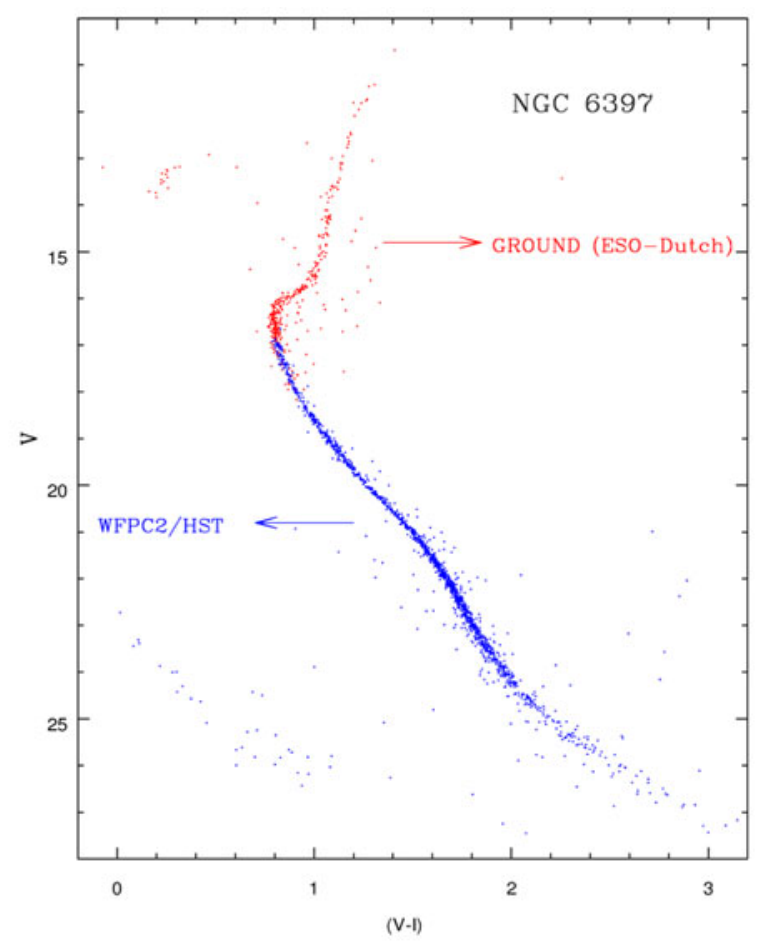

Figure 1. The CMD of NGC 6397 shows very narrow sequences and a well defined TO, supporting the idea that, in general, GCs are populated by coeval and chemically homogeneous stars.

et al. 1999). This problem, usually known as the the second parameter problem, still lacks of a comprehensive understanding: many mechanisms, and many parameters have been proposed to explain the HB peculiarities, but none apparently is able to explain the entire observational scenario. It is well possible that a combination of parameters is responsible for the HB morphology (Fusi Pecci et al. 1993). Surely, the total cluster mass seems to have a relevant role (Recio-Blanco et al. 2006).

It is tempting to relate the second parameter problem to the complex abundance pattern of GCs. Since high Na and low O abundances are signatures of material processed through hot H-burning, they should be accompanied by high He-contents (D'Antona \& Caloi 2004). In most cases, small He excesses up to dY 0.04 (that is Y 0.28 , assuming the original He content was the Big Bang one) are expected. While this should have small impact on colors and magnitudes of stars up to the tip of the RGB, a large impact is expected on the colors of the HB stars, since He-rich stars should be less massive. E.g., in the case of GCs of intermediate metallicity ([Fe/H] -1.5$)$, the progeny of He-rich, Na-rich, O-poor RGB stars should reside on the blue part of the HB, while that of the "normal" He-poor, Na-poor, O-rich stars should be within the instability strip or redder than it. Actually mean HB colors are also influenced by small age differences of 2-3 Gyr. However, within a single GC a correlation is expected between the distribution of masses (i.e. colors) of the HB-stars and of $\mathrm{Na}$ and $\mathrm{O}$ abundances.

In summary, a number of apparently independent observational facts seems to suggest that, at least in some GCs, there are stars which have formed from material which must have been processed by a previous generation of stars. 

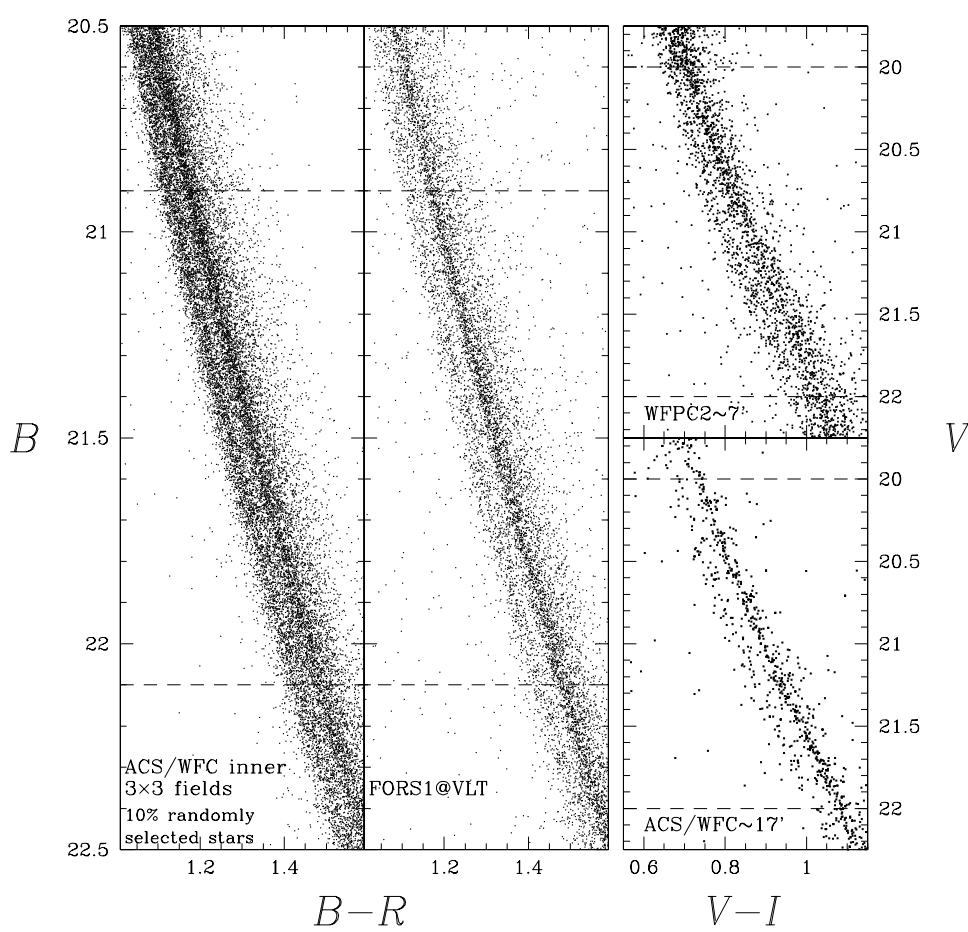

Figure 2. The double MS of Omega Centauri. The MS split is visible from the cluster center to its outskirts, though the ratio of the number of stars populating the two sequences decreases from the cluster core to its outer envelope (from Bellini et al. 2008, in preparation).

The questions is: do we have some direct, observational evidence of the presence of multiple populations in GCs? Very recent discoveries, made possible by high accuracy photometry on deep HST images, allowed us to positively answer to this question. In this paper, we will summarize these new observational facts, and briefly discuss their link to the complex abundance pattern and to the anomalous HBs.

\section{Direct Observational Evidence of Multiple Populations in GCs}

The first, direct observational evidence of the presence of more than one stellar population in a GCs was published by Bedin et al. (2004). Bedin et al. found that, for a few magnitudes below the turn-off (TO), the main sequence (MS) of $\omega$ Centauri splits in two (Fig. 2). Indeed, the suspect of a MS split in $\omega$ Cen was already raised by Jay Anderson in his PhD thesis, but the result was based on only one external WFPC2 field, and this finding was so unexpected that he decided to wait for more data and more accurate photometry to be sure of its reality. Indeed, Bedin et al. (2004) confirmed the MS split in Jay Anderson field and in an additional ACS field located 17 arcmin from the cluster center. Now, we know that the multiple MS is present all over the cluster, though the ratio of blue to red MS stars diminishes going from the cluster core to its envelope (Sollima et al. 2007, Bellini et al. 2008, in preparation).

The more shocking discovery on the multiple populations in $\omega$ Cen, however, came from a follow-up spectroscopic analysis that showed that the blue MS has twice the metal abundance of the dominant red branch of the MS (Piotto et al. 2005). The only isochrones 

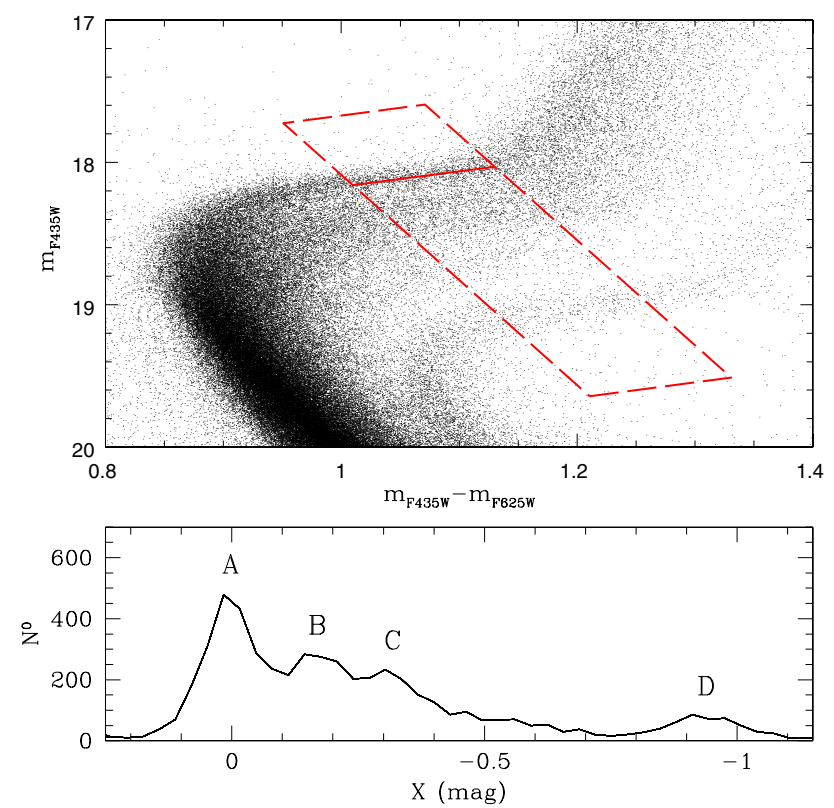

Figure 3. The multiple SGB in Omega Centauri. There are at least 4 distinct SGBs, plus a small fraction of stars spreaded between SGB-C and SGB-D (from Villanova et al. 2007).

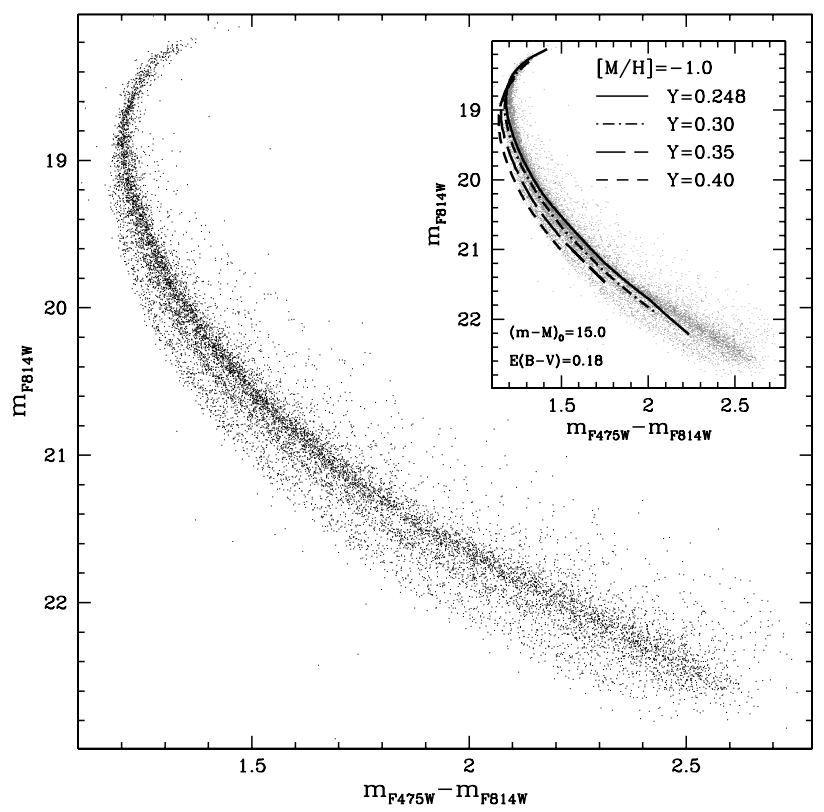

Figure 4. The spectacular triple MS of NGC 2808. Note the narrowness of the turnoff. The isochrone fitting in the inset shows that the bluest sequence can be reproduced only with a He content as high as $\mathrm{Y}=0.40$ (from Piotto et al. 2007). 
that would fit this combination of color and metallicity were extremely enriched in helium $(Y \sim 0.38)$ relative to the dominant old-population component, which presumably has primordial helium.

Indeed, the scenario in $\omega$ Cen is even more complex. As is already evident in the CMD of Bedin et al. (2004), this object has at least three MSs, which spread into a highly multiple sub-giant branch (SGB) with at least four distinct components (Fig. 3) characterized by different metallicities and ages (Sollima et al. 2005, Villanova et al. 2007; the latter has a detailed discussion.) A fifth, more dispersed component is spread between the SGB-C and SGB-D of Fig. 3.

These results reinforced the suspicion that the multiple MS of $\omega$ Cen could just be an additional peculiarity of an already anomalous object, which might not even be a GC, but a remnant of a dwarf galaxy instead. In order to shed more light on the possible presence of multiple MSs in Galactic GCs, we undertook an observational campaign with $H S T$, properly devised to search multiple sequences at the level of the upper-MS, turnoff (TO), and SGB. The new data allowed us to show that the multiple evolutionary sequence phenomenon is not a peculiarity of $\omega$ Centauri only.

As shown in Fig. 4, also the CMD of NGC 2808 is splitted into three MSs (Piotto et al. 2007). Because of the negligible dispersion in Fe peak elements (Carretta et al. 2006), Piotto et al. (2007) proposed the presence of three groups of stars in NGC 2808, with three different He contents, in order to explain the triple MS of Fig. 4. These groups may be associated to the three groups with different Oxygen content discovered by Carretta et al. (2006). These results are also consistent with the presence of a multiple HB, as discussed in D'Antona and Coloi (2004) and D'Antona et al. (2006). Finally, we note that the narrowness of the TO region displayed by Fig. 4 suggests that the three stellar populations of NGC 2808 must have a small age dispersion, much less than 1 Gyr.

Also NGC 1851 must have at least two, distinct stellar populations. In this case the observational evidence comes from the split of the SGB in the CMD (Fig. 5) of this cluster (Milone et al. 2007). Would the magnitude difference between the two SGBs be due only to an age difference, the two star formation episodes should have been separated by at least 1 Gyr. However, as shown by Cassisi et al. (2007), the presence in NGC 1851 of two stellar populations, one with a normal $\alpha$-enhanced chemical composition, and one characterized by a strong CNONa anticorrelation pattern could reproduce the observed CMD split. In this case, the age spread between the two populations could be much smaller, possibly consistent with the small age spread implied by the narrow TO of NGC 2808. In other terms, the SGB split would be mainly a consequence of the metallicity difference, and only negligibly affected by (a small) age dispersion. Cassisi et al. (2007) hypothesis is supported by the presence of a group of CN-strong and a group of CN-weak stars discovered by Hesser et al. 1982, and by a recent work by Yong and Grundahl (2007) who find a $\mathrm{NaO}$ anticorrelation among NGC 1851 giants.

NGC 1851 is considered a sort of prototype of bimodal HB clusters. Milone et al. (2007) note that the fraction of fainter/brighter SGB stars is remarkably similar to the fraction of bluer/redder HB stars. Therefore, it is tempting to associate the brighter SGB stars to the CN-normal, s-process element normal stars and to the red HB, while the fainter SGB should be populated by CN-strong, s-process element-enhanced stars which should evolve into the blue HB. In this scenario, the faint SGB stars should be slightly younger (by a few $10^{7}$ to a few $10^{8}$ years) and should come from processed material which might also be moderately He enriched, a fact that would help explaining why they evolve into the blue HB. By studying the cluster MS, Milone et al. (2007) exclude an He enrichment larger than $\Delta Y=0.03$ ), as expected also by the models of Cassisi et al. (2007). Nevertheless, this small He enrichment, coupled with an enhanced mass loss, would be sufficient to move 


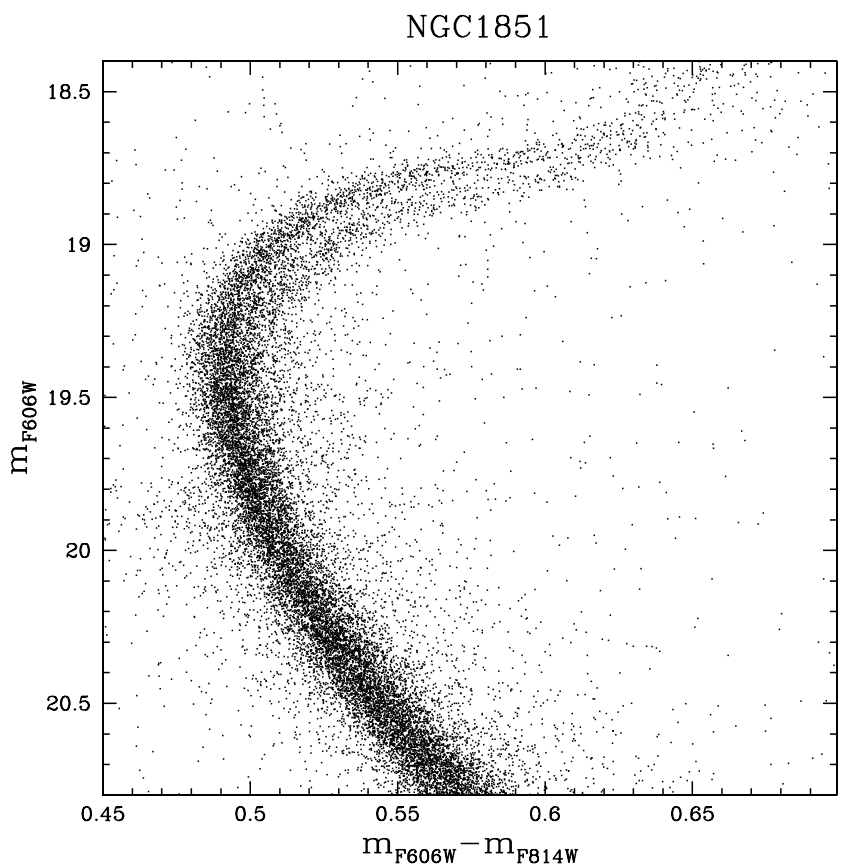

Figure 5. The double SGB in NGC 1851. The two SGBs are separated by about 0.12 magnitudes in F606W (from Milone et al. 2007)

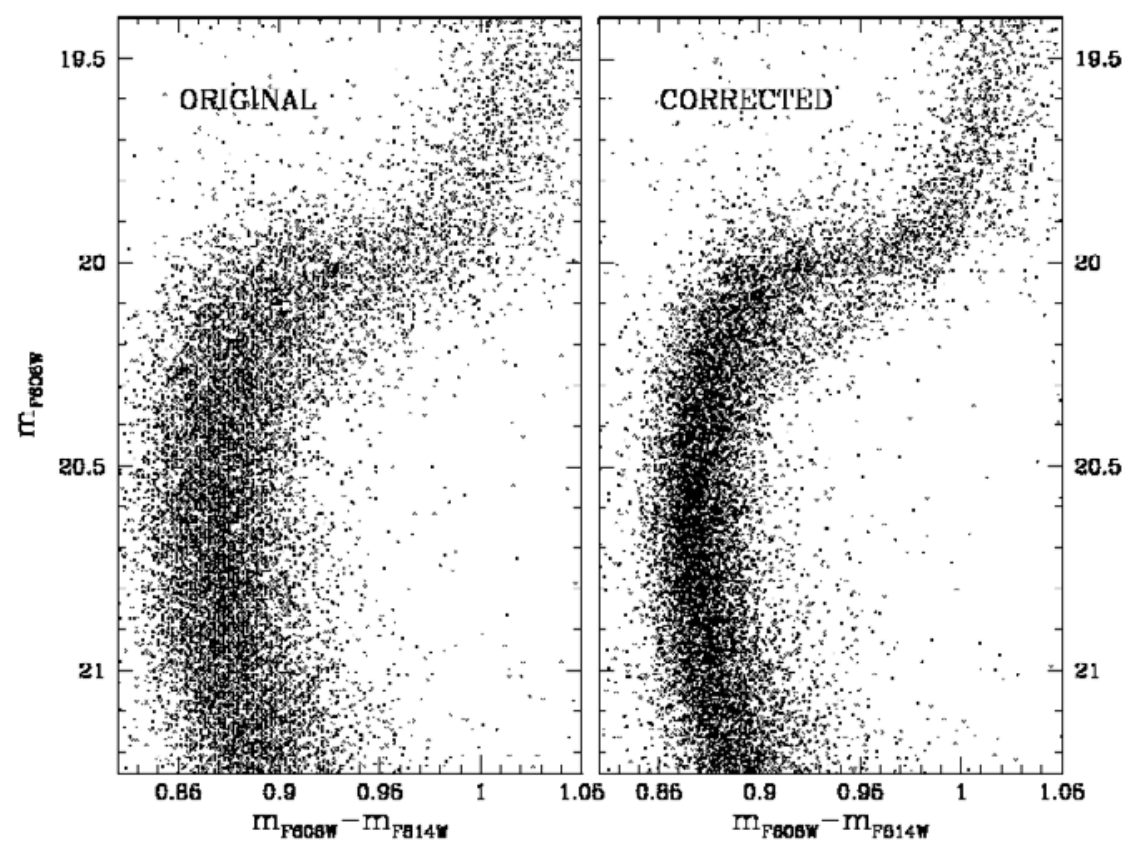

Figure 6. After the correction for differential reddening, also NGC 6388 shows a double SGB (Piotto et al. 2008, in preparation). 
stars from the red to the blue side of the RR Lyrae instability strip. Direct spectroscopic measurements of the SGB and HB stars in NGC 1851 are badly needed.

There is at least another cluster which undoubtedly shows a split in the SGB: NGC 6388 (Piotto et al. 2008, in preparation). Figure 6 shows that, even after correction for differential reddening, the SGB of NGC 6388 closely resembles the SGB of NGC 1851. NGC 6388, as well as its twin cluster NGC 6441, are two extremely peculiar clusters. Since Rich et al. (1997), we know that, despite their high metal content, higher than in 47 Tucanae, they have a bimodal HB, which extends to extremely hot temperatures (Busso et al. 2007), totally un-expected for this metal rich cluster. NGC 6388 stars also display a $\mathrm{NaO}$ anticorrelation (Carretta et al. 2007). Unfortunately, available data do not allow us to study the MS of this cluster, searching for a MS split. Hopefully, new data coming from the HST program GO11233 should help to constrain the MS width, and therefore the He dispersion. In this context, it is worth noting that Caloi and D'Antona (2007), in order to reproduce the HB of NGC 6441, propose the presence of three populations, with three different He contents, one with an extreme He enhancement of $Y=0.40$. Such a strong enhancement should be visible in a MS split, as in the case of $\omega$ Cen and NGC 2808. A strong He enhancement and a consequent MS split may also apply to NGC 6388, because of the many similitudes with NGC 6441 ..

One more cluster, M54, shows a complex CMD (see, e.g., Siegel et al. 2007). This cluster has been shown, however, in too many papers to cite here, to be a part of the Sagittarius dwarf galaxy that is in process of merging into the Milky Way, and very possibly the actual nucleus of that galaxy. Actually, it is still matter of debate which parts of the CMD of M54 represent the cluster population and which ones are due to the Sagittarius stars. M54 may have a complex stellar populations as $\omega$ Cen, though this fact will be much harder to demonstrate.

Finally, we note that the multiple population phenomenon in star clusters may not be confined only to Galactic GCs. Mackey \& Broby Nielsen (2007) suggest the presence of two populations with an age difference of $\sim 300 \mathrm{Myr}$ in the 2 Gyr old cluster NGC 1846 of the Large Magellanic Cloud (LMC). In this case, the presence of the two populations is inferred by the presence of two TOs in the CMD (Fig. 7). These two populations may either be the consequence of a tidal capture of two clusters or NGC 1846 may be showing something analogous to the multiple populations identified in the Galactic GCs. NGC 1846 might not be an exception among LMC clusters. Vallenari et al. (1994) already suggested the possibility of the presence of two stellar populations in the LMC cluster NGC 1850. A quick analysis of the CMDs of about 50 clusters from ACS/HST images shows that about $10 \%$ of them might show evidence of multiple generations (Milone $\mathrm{et}$ al. 2008, in preparation).

\section{Discussion}

So far, we have identified four Galactic globular clusters for which we have a direct evidence of multiple stellar populations, and they are all quite different:

(a) In $\omega$ Centauri $\left(\sim 4 \times 10^{6} M_{\odot}\right)$, the different populations manifest themselves both in a MS split (interpreted as a split in He and metallicity abundances) and in a SGB split (interpreted in terms of $\mathrm{He}$, metallicity, and age variations $>1 \mathrm{Gyr}$ ) which implies at least four different stellar groups within the same cluster, which formed in a time interval greater than 1 Gyr. Omega Centauri has also a very extended HB (EHB), which extends far beyond $30.000 \mathrm{~K}$.

(b) In NGC $2808\left(\sim 1.6 \times 10^{6} M_{\odot}\right)$, the multiple generation of stars is inferred from the presence of three MSs (also in this case interpreted in terms of three groups of stars, 


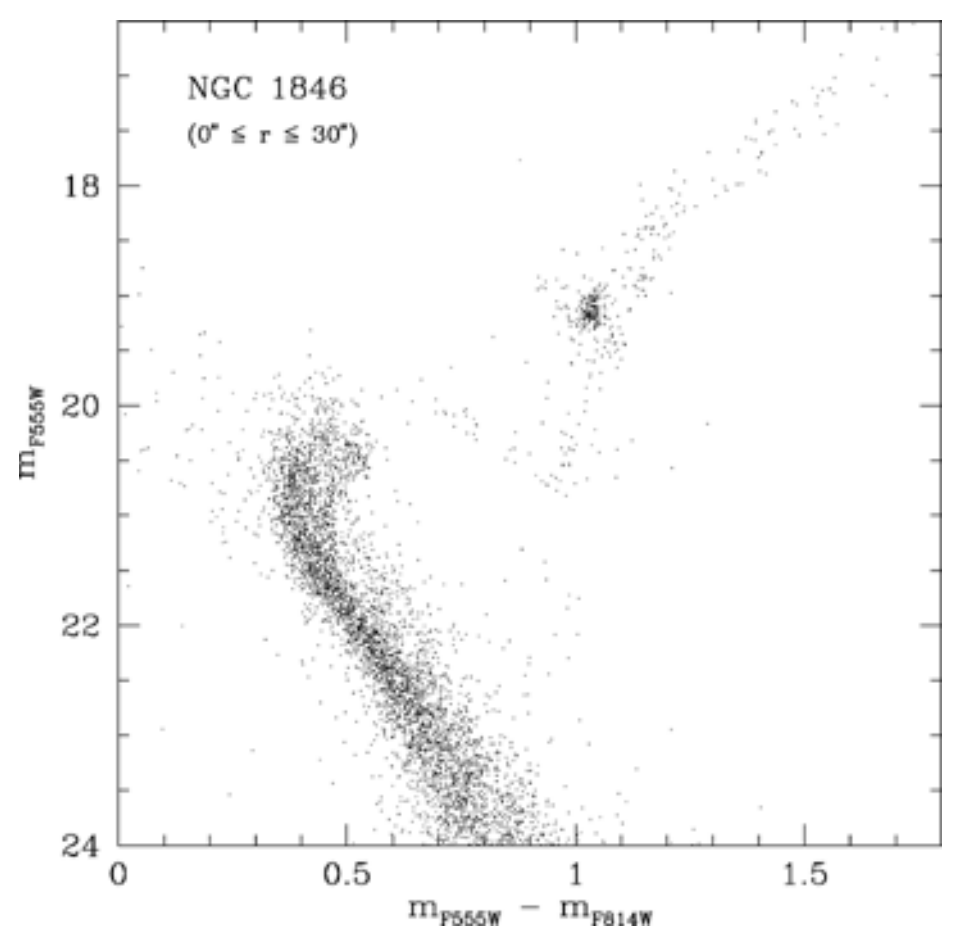

Figure 7. The double main sequence turn off of the Large Magellanic Cloud cluster NGC 1846 (from Mackey \& Broby Nielsen 2007).

with different He content), possibly linked to three stellar groups with different oxygen abundances, and possibly to the multiple HB. The age difference between the 3 groups is significantly smaller than 1 Gyr. It also has an EHB, extended as much as the $\mathrm{HB}$ of $\omega$ Cen. It shows an extended $\mathrm{NaO}$ anticorrelation.

(c) In the case of NGC $1851\left(\sim 1.0 \times 10^{6} M_{\odot}\right)$, we have evidence of two stellar groups from the SGB split. It is difficult to establish the age difference between the two stellar populations without a detailed chemical abundance analysis. However, available observational evidence seems to imply that the SGB split may be due to a difference in $\mathrm{CN}, \mathrm{Na}, \mathrm{O}$, and s-process elements, while the age difference could be small (e.g. as small as in the case of NGC 2808). If the SGB split would be due only to age, the two star formation episodhes should have been happened with a time separation of $\sim 1$ Gyr. From the analysis of the cluster CMD, there seems to be no MS split, which would imply a small He spread, if any $(\Delta Y<0.03)$. The cluster has no EHB, but it shows a bimodal HB. It shows a $\mathrm{NaO}$ anticorrelation.

(d) In NGC $6388\left(\sim 1.6 \times 10^{6} M_{\odot}\right)$ we have evidence of two stellar groups from a SGB split. With the available observational data it is not possible to establish whether there is a split in the MS of this GC. NGC 6388 has an EHB, possibly as extended as in the cases of NGC 2808 and $\omega$ Cen. It shows an extended $\mathrm{NaO}$ anticorrelation.

Another massive $\left(\sim 2.0 \times 10^{6} M_{\odot}\right)$ GC, M54, is suspected to host multiple populations, though the analysis is strongly hampered by the contamination of the Sagittarius galaxy. Also M54 has an EHB, similar to the HB of NGC 2808 and $\omega$ Cen (Rosenberg et al. 2004).

At least one LMC intermediate age cluster shows a population split at the level of the TO: NGC 1846. This is a massive clusters, among the most massive LMC clusters 
according to Chrysovergis et al. (1999), though probably not as massive as the above Galactic GCs (a more accurate mass estimate for this cluster is needed). Other LMC clusters are suspected to shows a similar TO splitting.

Many GCs are clearly not simple, single-stellar-population objects. The emerging evidence is that the star-formation history can vary strongly from GC to GC, and that, GCs are able to produce very unusual objects, as no such He-rich MS stars have ever been found elsewhere. At the moment, we can note that the three GCs in which multiple generations of stars have been clearly identified (Omega Cen, NGC 2808, and NGC 1851), and the two other GCs suspected to contain more than one stellar generation (NGC 6388 and NGC 6441: Fig 6, Caloi \& D'Antona 2007, Busso et al. 2007) are among the ten most massive GCs in our Galaxy. This evidence suggests that cluster mass might have a role in the star-formation history of GCs.

Reconstruction of this star-formation history requires a a better understanding of the chemical enrichment mechanisms, but the site of hot H-burning remains unclear. There are two requisites: (i) temperature should be high enough; and (ii) the stars where the burning occur should be able to give back the processed material to the intracluster matter at a velocity low enough that it can be kept within the GC itself (a few tens of $\mathrm{km} / \mathrm{s}$ ). Candidates include: (i) Massive $\left(M>10 M_{\odot}\right)$ rotating stars (Decressin et al. 2007); (ii) the most massive among the intermediate mass stars undergoing hot bottom burning during their AGB phase (Ventura et al. 2001). The two mechanisms act on different timescales $\left(10^{7}\right.$ and $10^{8} \mathrm{yr}$, respectively), and both solutions have their pros and cons. The massive star scenario should avoid mixture of O-poor, Na-rich material with that rich in heavy elements from SNe, while it is not clear how the chemically processed material could be retained by the proto-cluster in spite of the fast winds and SN explosions always associated to massive stars. Producing the right pattern of abundances from massive AGB stars seems to require considerable fine tuning. In addition, both scenarios require that either the IMF of GCs was very heavily weighted toward massive stars, or that some GCs should have lost a major fraction of their original population (Bekki and Norris 2006), and then may even be the remnants of tidally disrupted dwarf galaxies, as suggested by the complexity in the CMD of $\omega$ Cen and M54.

The observational scenario is becoming more complex, but, the new results might have indicated the right track for a comprehensive understanding of the formation and early evolution of GCs. We are perhaps for the first time close to compose what has been for decades and still is a broken puzzle.

Acknowledgements. I wish to warmly thank J. Anderson, Andrea Bellini, Luigi R. Bedin, Ivan R. King, Antonino P. Milone, without whom most of the results presented in this review would not have been possible. I wish also to thank Sandro Villanova for his help in the spectroscopic investigation of Omega Centauri main sequence and turn off stars. A special thanks to Alvio Renzini and Raffaele Gratton for the many enthusiastic discussions on the subject of multipopulations in globular clusters.

\section{References}

Bedin, L. R., Piotto, G., Anderson, J., Cassisi, S., King, I. R., Momany, Y., \& Carraro, G. 2004, ApJ (Letters), 605, L125

Bekki, K. \& Norris, J. E. 2006, ApJ (Letters), 637, L109

Busso et al. (2007), A\&SA, 474, 105

Caloi, V. \& D'Antona, F. 2007, A\&A, 463, 949

Carretta, E., Bragaglia, A., Gratton, R. G., Leone, F., Recio-Blanco, A., \& Lucatello, S. 2006, $A \mathscr{E} A, 450,523$ 
Carretta, E. et al. 2007, A\&A, 464, 957

Cassisi, S., Salaris, M., Pietrinferni, A., Piotto, G., Milone, A. P., Bedin, L. R., \& Anderson, J. 2007, arXiv0711.3823

Charbonnel, C. 1994, A\&A, 282, 811

Chrysovergis, M., Kontizas, M., \& Kontizas, E. 1989, A 6 AS, 77, 357

Cohen, J. G., Briley, M. M., \& Stetson, P. B. 2002, AJ, 123, 2525

D'Antona, F. \& Caloi, V. 2004, ApJ, 611, 871

D'Antona, F., Bellazzini, M., Caloi, V., Pecci, F. Fusi, Galleti, S., \& Rood, R. T. 2006, ApJ, 631,868

Decressin, T., Meynet, G., Charbonnel, C., Prantzos, N., \& Ekström, S. 2007, A\&AA, 464, 1029

Ferraro, F. R., Paltrinieri, B., Fusi Pecci, F., Rood, R. T., \& Dorman, B. ApJ, 500, 311

Fusi Pecci, F., Ferraro, F. R., Bellazzini, M., Djorgovski, S., Piotto, G., \& Buonanno, R. 1993, $A J, 105,1145$

Gratton, R., Sneden, C., Carretta, E., \& Bragaglia, A., A $\& A, 354,169$

Gratton, R. et al. 2001, A\& $A, 369,87$

Gratton, R., Sneden, C., \& Carretta, E. 2004, ARAA, 42, 385

Hesser, J. E., Bell, R. A., Harris, G. L. H., \& Cannon, R. D. 1982, AJ, 87, 1470

Mackey, A. D. \& Broby Nielsen, P. 2007, MNRAS, 379, 151

Milone, A. P. et al. 2007, in press, arXiv0709.3762

Piotto, G., Zoccali, M., King, I. R., Djorgovski, S. G., Sosin, C., Rich, R. M., \& Meylan, G. 1999, AJ, 118, 1727

Piotto, G., et al. 2005, ApJ, 621, 777 (P05)

Piotto, G., et al. 2007, ApJ (Letters), 661, L53 (P07)

Prantzos, N., Charbonnel, C., \& Iliadis, C. 2007, A\& $A, 470,179$

Recio-Blanco, A., Aparicio, A., Piotto, G., de Angeli, F., \& Djorgovski, S. G. 2006, A\&A, 452, 875

Rich, R. M., Sosin, C., Djorgovski, S. G., Piotto, G., King, I. R., Renzini, A., Phinney, E. S., Dorman, B., Liebert, J., \& Meylan, G., 1997, ApJ (Letters), 484, L25

Rosenberg, A., Recio-Blanco, A.,\& García-Marn, M. 2004, ApJ, 603, 135

Sandage, A. \& Wildey, R. 1967, ApJ, 150, 469

Siegel et al. 2007, ApJ (Letters), 667, L57

Sollima, A., Pancino, E., Ferraro, F. R., Bellazzini, M., Straniero, O., \& Pasquini, L. 2005, ApJ, 634, 332

Sollima, A., Ferraro, F. R., Bellazzini, M., Origlia, L., Straniero, O., \& Pancino, E. 2007, ApJ, 654,915

Sweigart, A. V., \& Mengel, J. G. 1979, ApJ, 229, 624

Ventura, P., D'Antona, F., Mazzitelli, I., \& Gratton, R. 2001, ApJ (Letters), 550, L65

Vallenari, A., Aparicio, A., Fagotto, F., Chiosi, C., Ortolani, S., \& Meylan, G. 1994, A\&A, 284, 447

Villanova, S., et al. 2007, ApJ, 663, 296

van den Bergh, S. AJ, 72, 70

Yong, D. \& Grundahl, F. 2007, arXiv0711.1394 